\title{
ENTRANCE REGION FLOW IN CONCENTRIC ANNULI WITH ROTATING INNER WALL FOR BINGHAM FLUID
}

\author{
SRinivasa RaO Nadiminti ${ }^{1}$ And A. Kandasamy ${ }^{2}$ \\ Department of Mathematical and Computational Sciences \\ National Institute of Technology Karnataka, Surathkal, Mangalore - 575025, India \\ ${ }^{1}$ srinudm@gmail.com ${ }^{2}$ kandy@nitk.ac.in
}

[Received: February 25, 2016, Accepted: July 28, 2016]

\begin{abstract}
Entrance region flow in concentric annuli with rotating inner wall for Bingham non-Newtonian fluid has been studied numerically. The inner cylinder is assumed to be rotating with a constant angular velocity $\omega$ and the outer cylinder to be stationary. Finite difference analysis is used to obtain the velocity components $U, V, W$ and the distribution of pressure $P$ along the radial direction $R$. With Prandtl's boundary layer assumptions, the continuity and momentum equations are solved iteratively using a finite difference method. Computational results are obtained for various non-Newtonian flow parameters and geometrical considerations. The development of the axial velocity profile, radial velocity profile, tangential velocity profile and pressure distribution in the entrance region have been analyzed. Comparison of the present results with the results available in literature for various particular cases has been done and found to be in agreement.
\end{abstract}

Mathematical Subject Classification: $76 \mathrm{U} 05$

Keywords: Concentric annuli, Bingham fluid, entrance region flow, finite difference method, rotating wall

\section{NOMENCLATURE}

$k$

$m$

$p$

$p_{0}$

$P$

$r$

$z$

$\theta$

$R=r / R_{2}$

$Z=2 z(1-N) /\left(R_{2} R e\right)$

$R_{1}$

$R_{2}$

$B=\tau_{0} R_{2} /\left(k u_{0}\right)$

$R e=2 \rho\left(R_{2}-R_{1}\right) u_{0} / k$ consistency index (Pa.s),

number of radial increments in the numerical mesh net-

work,

pressure $(P a)$,

initial pressure $(P a)$,

dimensionless pressure, $\left(p-p_{0}\right) /\left(\rho u_{0}^{2}\right)$,

radial coordinate $(m)$,

axial coordinate $(m)$,

tangential coordinate ( $\mathrm{rad})$,

dimensionless coordinate in the radial direction,

dimensionless coordinate in the axial direction,

radius of the inner cylinder $(m)$,

radius of the outer cylinder $(m)$,

Bingham number,

Reynolds number, 


\begin{tabular}{ll}
\hline$T_{a}$ & Taylor number \\
$N=R_{1} / R_{2}$ & dimensionless aspect ratio of the annulus, \\
$u$ & velocity components in $z$ direction $(\mathrm{m} / \mathrm{s})$, \\
$v$ & velocity components in $r$ direction $(\mathrm{m} / \mathrm{s})$, \\
$w$ & velocity components in $\theta$ direction $(\mathrm{m} / \mathrm{s})$, \\
$u_{0}$ & uniform inlet velocity $(\mathrm{m} / \mathrm{s})$, \\
$U=u / u_{0}$ & dimensionless velocity component in z direction, \\
$V=\rho v R_{2} / \mu_{r}$ & dimensionless velocity component in $\mathrm{r}$ direction, \\
$W=w /\left(\omega R_{1}\right)$ & dimensionless velocity component in $\theta$ direction, \\
$\rho$ & density of the fluid $\left(\mathrm{kg} / \mathrm{m}^{3}\right)$, \\
$\mu$ & apparent viscosity of the model $(P a . s)$, \\
$\tau_{0}$ & yield stress $(P a)$, \\
$\omega$ & regular angular velocity $(\mathrm{rad} / \mathrm{s})$, \\
$\Delta R$ & dimensionless mesh size in the radial direction, \\
$\Delta Z$ & dimensionless mesh size in the axial direction.
\end{tabular}

\section{INTRODUCTION}

The problem of entrance region flow in concentric annuli with rotating inner wall for non-Newtonian fluids is of practical importance in engineering applications such as the design of cooling systems for electric machines, compact rotary heat exchangers and combustion chambers, axial-flow turbo machinery and polymer processing industries. In the nuclear reactor field, laminar flow conditions occur when the coolant flow rates are reduced during periods of low power operation. Many important industrial fluids are non-Newtonian in their flow characteristics and are referred to as rheological fluids. These include blood, various suspensions such as coal-water or coal-oil slurries, glues, inks, foods, polymer solutions, paints and many others. The fluid considered here is the Bingham model, which is of the 'time-independent yield stress' fluid category.

The problem of entrance region flow of non-Newtonian fluids in an annular cylinders has been studied by various authors. Mishra et al. [1] studied the flow of the Bingham plastic fluids in the concentric annulus and obtained results for boundary layer thickness, centre core velocity, pressure distribution. Batra and Bigyani Das [2] developed the stress-strain relation for the Casson fluid in the annular space between two coaxial rotating cylinders where the inner cylinder is at rest and outer cylinder is rotating. Maia and Gasparetto 3] applied a finite difference method for the Power-law fluid in the annuli and found differences in the entrance geometries. Sayed-Ahmed and Hazem [4] applied a finite difference method to study the laminar flow of a Power-Law fluid in the concentric annuli with rotating inner wall. Recently, Kandasamy and Srinivasa Rao [5] investigated entrance region flow in concentric annuli with a rotating inner wall for Herschel-Bulkley Fluids. The constitutive equation for Bingham fluid is given by Bird et al. 6]

$$
\tau_{i j}=\left(\mu+\frac{\tau_{0}}{\varepsilon}\right) \varepsilon_{i j} \quad\left(\tau \geq \tau_{0}\right)
$$


where

$$
\tau=\sqrt{\frac{1}{2} \tau_{i j} \tau_{i j}} \quad \text { and } \quad \varepsilon=\sqrt{\frac{1}{2} \varepsilon_{i j} \varepsilon_{i j}}
$$

where $\tau_{0}$ is the yield stress, $\tau_{i j}$ and $\varepsilon_{i j}$ are the stress tensor and the rate-of-strain tensor, respectively. and $\mu$ is the viscosity of the fluid.

Moreover, Kandasamy [7 investigated the entrance region flow heat transfer in concentric annuli for a Bingham fluid and presents the velocity distributions, temperature and pressure in the entrance region. Round and $\mathrm{Yu}[8]$ analyzed the developing flows of Herschel-Bulkley fluids through concentric annuli. Flow of Casson fluid in a narrow tube with a side branch was investigated by Misra and Ghosh 9. Flow of Casson fluid in a pipe filled with a homogeneous porous medium has been considered by Dash et al. [10]. Ahmed and Attia [1] investigated Magneto hydrodynamic flow and heat transfer of a non-Newtonian fluid in an eccentric annulus. An analytical solution for the entrance region blood flow in a concentric annuli was obtained by Batra and Jena [12] assuming blood to obey Casson model.

Further, Manglik and Fang 13 numerically investigated the flow of non-Newtonian fluids through annuli. Nouar et al. 14] reported the results of numerical analysis of the thermal convection for Herschel-Bulkley fluids. Poole and Chhabra [15] reported the results of a systematic numerical investigation of developing laminar pipe flow of yield stress fluids. Entropy generation in Non-Newtonian fluids due to heat and mass transfer in the entrance region of ducts was investigated by Galanis and Rashidi [16. Rhashidi et al. [17. studied the investigation of heat transfer in a porous annulus with pulsating pressure gradient by the homotopy analysis method. Recently, Rekha and Kandasamy [18] investigated the entrance region flow of Bingham fluid in an annular cylinder.

In the present work, the problem of entrance region flow of Bingham fluid in concentric annuli has been investigated. The analysis has been carried out under the assumption that the inner cylinder is rotating and the outer cylinder is at rest. With Prandtl's boundary layer assumptions, the equation of conservation of mass and momentum are discretized and solved using linearized implicit finite difference technique. The system of linear algebraic equations thus obtained has been solved by the GaussJordan method. The development of axial velocity profile, radial velocity profile, tangential velocity profile and pressure distribution in the entrance region have been determined for different values of non-Newtonian flow characteristics and geometrical parameters. The effects of these on the velocity profiles and pressure distribution are discussed.

\section{Formulation of the PROBlem}

The geometry of the problem is shown in Figure 1. The Bingham fluid enters the horizontal concentric annuli with inner and outer radii $R_{1}$ and $R_{2}$, respectively, from a large chamber with a uniform flat velocity profile $u_{0}$ along the axial direction $z$ and with an initial pressure $p_{0}$. The inner cylinder rotates with an angular velocity $\omega$ and the outer cylinder is at rest. The flow is steady, laminar, incompressible, axisymmetric 


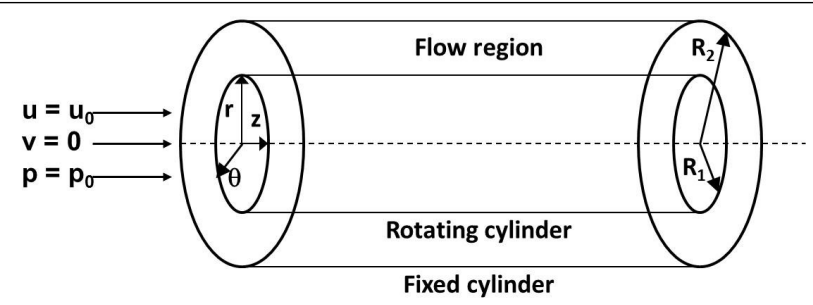

Figure 1. Geometry of the Problem

and of constant physical properties. We consider a cylindrical polar coordinate system with the origin at the inlet section on the central axis of the annulus, the $z$-axis along the axial direction and the radial direction $r$ perpendicular to the $z$-axis. Under the above assumptions with the usual Prandtl's boundary layer assumptions [19, the governing equations in a polar coordinate system $(r, \theta, z)$ for a Bingham fluid in the entrance region are:

Continuity equation : $\frac{\partial(r v)}{\partial r}+\frac{\partial(r u)}{\partial z}=0$

$r-$ momentum equation $: \frac{w^{2}}{r}=\frac{1}{\rho} \frac{\partial p}{\partial r}$

$\theta-$ momentum equation $: v \frac{\partial w}{\partial r}+u \frac{\partial w}{\partial z}+\frac{v w}{r}=\frac{1}{\rho r^{2}} \frac{\partial}{\partial r}\left(r^{2}\left[\tau_{0}+k r \frac{\partial}{\partial r}\left(\frac{w}{r}\right)\right]\right)$

$z-$ momentum equation $: v \frac{\partial u}{\partial r}+u \frac{\partial u}{\partial z}=-\frac{1}{\rho} \frac{\partial p}{\partial z}+\frac{1}{\rho r} \frac{\partial}{\partial r}\left(r\left[\tau_{0}+k \frac{\partial u}{\partial r}\right]\right)$

where $u, v, w$ are the velocity components in the directions $z, r, \theta$, respectively, $\rho$ is the density of the fluid, $k$ is the consistency index and $p$ is the pressure.

The boundary conditions of the problem are given by

$$
\begin{aligned}
& \text { for } z \geq 0 \text { and } r=R_{1}, v=u=0 \text { and } w=\omega R_{1}, \\
& \text { for } z \geq 0 \text { and } r=R_{2}, v=u=w=0, \\
& \text { for } z=0 \text { and } R_{1}<r<R_{2}, u=u_{0}, \\
& \text { at } z=0, p=p_{0} .
\end{aligned}
$$

Using the boundary conditions 2.5, the continuity equation 2.1 can be expressed in the following integral form:

$$
2 \int_{R_{1}}^{R_{2}} r u \mathrm{~d} r=\left(R_{2}^{2}-R_{1}^{2}\right) u_{0}
$$


It is worth introducing the following dimensionless variables and parameters:

$$
\begin{gathered}
R=\frac{r}{R_{2}}, \quad U=\frac{u}{u_{0}}, \quad V=\frac{\rho v R_{2}}{\mu_{r}}, \\
W=\frac{w}{\omega R_{1}}, \quad N=\frac{R_{1}}{R_{2}}, \quad P=\frac{p-p_{0}}{\rho u_{0}^{2}}, \quad Z=\frac{2 z(1-N)}{R_{2} R e}, \\
B=\frac{\tau_{0} R_{2}}{k u_{0}}, \quad R e=\frac{2 \rho\left(R_{2}-R_{1}\right) u_{0}}{k}, \quad \mu_{r}=k\left(\frac{\omega R_{1}}{R_{2}}\right), \quad T_{a}=\frac{2 \omega^{2} \rho^{2} R_{1}^{2}\left(R_{2}-R_{1}\right)^{3}}{\mu_{r}^{2}\left(R_{1}+R_{2}\right)} .
\end{gathered}
$$

Here $B$ is the Bingham number, $R e$ is the Reynolds number, $T_{a}$ is the Taylor number, $\mu_{r}$ is known as reference viscosity and $N$ is the aspect ratio of the annulus.

Equations 2.1 to 2.4 and 2.6 in the dimensionless form are given by

$$
\begin{gathered}
\frac{\partial V}{\partial R}+\frac{V}{R}+\frac{\partial U}{\partial Z}=0 \\
\frac{W^{2}}{R}=\frac{R e^{2}(1-N)}{2(1+N) T_{a}} \frac{\partial P}{\partial R} \\
V \frac{\partial W}{\partial R}+U \frac{\partial W}{\partial Z}+\frac{V W}{R}=\frac{\partial^{2} W}{\partial R^{2}}+\frac{1}{R} \frac{\partial W}{\partial R}-\frac{W}{R^{2}}+\frac{2 B}{R} \\
V \frac{\partial U}{\partial R}+U \frac{\partial U}{\partial Z}=-\frac{\partial P}{\partial Z}+\frac{1}{R} \cdot \frac{\partial U}{\partial R}+\frac{\partial^{2} U}{\partial R^{2}}+\frac{B}{R}
\end{gathered}
$$

and

$$
2 \int_{N}^{1} R U \mathrm{~d} R=\left(1-N^{2}\right)
$$

The boundary conditions 2.5 in the dimensionless form are:

$$
\begin{aligned}
& \text { for } Z \geq 0 \text { and } R=N, \quad V=U=0 \text { and } W=1, \\
& \text { for } Z \geq 0 \text { and } R=1, \quad V=U=W=0, \\
& \text { for } Z=0 \text { and } N<R<1, \quad U=1, \\
& \text { at } Z=0, \quad P=0 .
\end{aligned}
$$

\section{Numerical SOlution}

The numerical analysis and the method of solution adopted here can be considered as an indirect extension of the work of Coney and El-Shaarawi [20. Considering the mesh network of Figure 2, the following difference representations are made. Here $\Delta R$ and $\Delta Z$ represent the grid size along the radial and axial directions, respectively.

$$
\begin{aligned}
& V_{i+1, j+1}= V_{i, j+1}\left(\frac{N+i \Delta R}{N+(i+1) \Delta R}\right)- \\
&-\frac{\Delta R}{4 \Delta Z}\left(\frac{2 N+(2 i+1) \Delta R}{N+(i+1) \Delta R}\right)\left(U_{i+1, j+1}+U_{i, j+1}-U_{i+1, j}-U_{i, j}\right) \\
& \frac{W_{i, j+1}^{2}}{N+i \Delta R}=\frac{(1-N) R e^{2}}{2 T_{a}(1+N)} \frac{P_{i, j+1}-P_{i-1, j+1}}{\Delta R}
\end{aligned}
$$




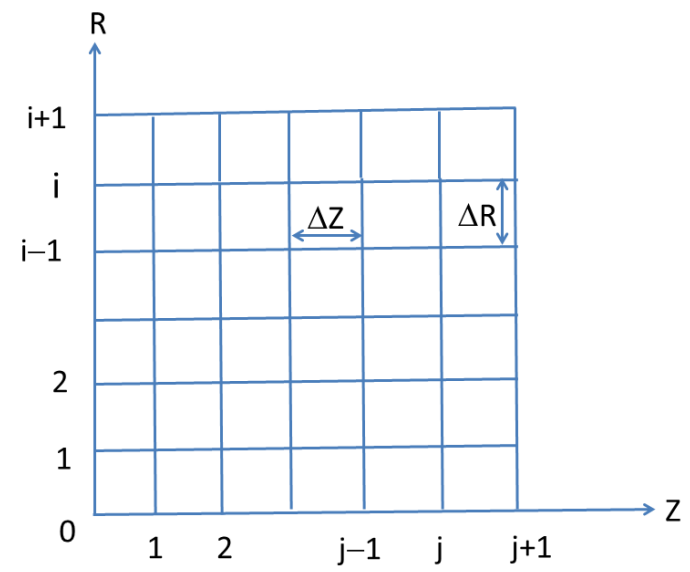

Figure 2. Grid formation for finite difference representations

$$
\begin{aligned}
& V i, j\left[\frac{W_{i+1, j+1}+W_{i+1, j}-W_{i-1, j}-W_{i-1, j+1}}{4 \Delta R}\right]+U i, j\left[\frac{W_{i, j+1}-W_{i, j}}{\Delta Z}\right]+\frac{V_{i, j} W_{i, j}}{N+i \Delta R}= \\
& =\frac{W_{i+1, j+1}+W_{i+1, j}-2 W_{i, j+1}-2 W_{i, j}+W_{i-1, j}+W_{i-1, j+1}}{2(\Delta R)^{2}}+ \\
& \quad+\frac{W_{i+1, j+1}+W_{i+1, j}-W_{i-1, j}-W_{i-1, j+1}}{(N+i \Delta R) 4 \Delta R}-\frac{W_{i j}}{(N+i \Delta R)^{2}}+\frac{2 B}{N+i \Delta R} \quad(3.3) \\
& V i, j\left[\frac{U_{i+1, j+1}-U_{i-1, j+1}}{2 \Delta R}\right]+U i, j\left[\frac{U_{i, j+1}-U_{i, j}}{\Delta Z}\right]= \\
& =-\frac{P_{i, j+1}-P_{i, j}}{\Delta Z}+\frac{U_{i+1, j+1}-U_{i-1, j+1}}{(N+i \Delta R) 2 \Delta R}+\frac{U_{i+1, j+1}-2 U_{i, j+1}+U_{i-1, j+1}}{(\Delta R)^{2}}+\frac{B}{N+i \Delta R}
\end{aligned}
$$

where $i=0$ at $R=N$ and $i=m$ at $R=1$.

The application of the trapezoidal rule to equation 2.11) yields

$$
\frac{\Delta R}{2}\left(N U_{0, j}+U_{m, j}\right)+\Delta R \sum_{i=1}^{m-1} U_{i, j}(N+i \Delta R)=\left(\frac{1-N^{2}}{2}\right)
$$

The boundary condition 2.12 gives $U_{0, j}=U_{m, j}=0$ and then the above equation reduces to

$$
\Delta R \sum_{i=1}^{m-1} U_{i, j}(N+i \Delta R)=\left(\frac{1-N^{2}}{2}\right)
$$

The set of difference equations (3.1) to (3.5) have been solved by the iterative procedure. Starting at the column $j=0$ (annulus entrance) and applying equation (3.3) 
for $1 \leq i \leq m-1$, we get a system of linear algebraic equations. This system has been solved by using the Gauss-Jordan method to obtain the values of the velocity component $W$ in the second column $j=1$. Then applying equations (3.2) and (3.4) for $1 \leq i \leq m-1$ and equation (3.5), we get a system of linear equations. Again solving this system by Gauss-Jordan method, we obtain the values of the velocity component $U$ and the pressure $P$ in the second column $j=1$. Finally, the values of the velocity component $V$ in the second column $j=1$ are obtained from equation (3.1) by Gauss-Jordan method using the known values of $U$. Repeating this procedure, we can advance, column by column, along the axial direction of the annulus until the flow becomes axially and tangentially fully developed.

\section{Results And Discussion}

Numerical calculations have been performed for all admissible values of Bingham number $B$, aspect ratio $N$ and various parameters as shown in Table 1 . Here, the velocity profiles and pressure distribution along the radial direction during the rotation of the inner wall of the annuli are shown in Figures 3426.

Table 1. List of various parameters used

\begin{tabular}{|c|c|c|c|c|}
\hline \multicolumn{5}{|c|}{ Various values of parameters } \\
\hline $\begin{array}{c}\text { Aspect } \\
\text { Ratio } N\end{array}$ & $\begin{array}{c}\text { Radial } \\
\text { position } R\end{array}$ & $\begin{array}{c}\text { Axial } \\
\text { position } Z\end{array}$ & Rt & $\begin{array}{c}\text { Bingham } \\
\text { number } B\end{array}$ \\
\hline 0.3 & 0.1 & $0.02,0.03$ & 0,20 & $0,10,20,30$ \\
0.5 & 0.1 & $0.02,0.03$ & 0,20 & $0,10,20,30$ \\
0.8 & 0.05 & $0.02,0.03$ & 0,20 & $0,10,20,30$ \\
\hline
\end{tabular}

Figures 3 to 8 show the development of the tangential velocity profile component $W$ for $N=0.3,0.5,0.8$ at axial positions of $Z=0.02,0.03$ and for different values of Bingham numbers $B$. Here, the parameter $R t$, which is the ratio of Reynolds number to Taylor number, is fixed as 20 . The values of tangential velocity decrease from the inner wall to outer wall of the annulus. Also, it is found that with the increase of aspect ratio $N$, the tangential velocity profile increases. That is, the tangential velocity is higher when the gap of the annuli is small. Further, it is found that with the increase of Bingham number, the tangential velocity profile increases. This means the tangential velocity tends to increase for thick viscous fluids when the inner cylinder is rotating. From the computed results corresponding to various values of $R t$, it is observes that the effect of the parameter $R t$ is negligible for the tangential velocity.

Figures 9 to 14 show the development of the axial velocity profile component $U$ for $N=0.3,0.5,0.8$ at axial positions of $Z=0.02,0.03$ and for different values of the Bingham numbers $B$. The computation was done for various values of the parameter Rt to study the effect of rotation of inner cylinder. The values corresponding to $R t=0$ and 20 are depicted in these figures. It is found that the velocity component 


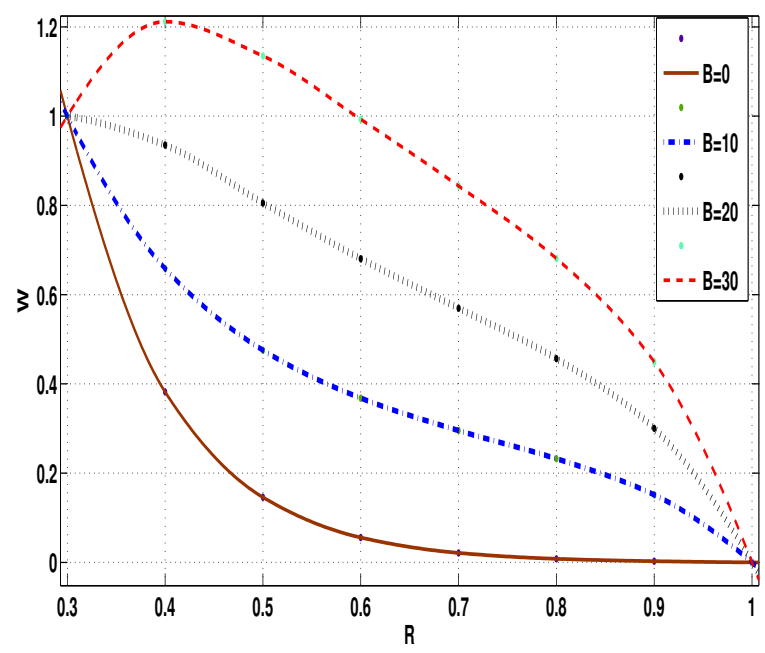

Figure 3. Tangential velocity profile for $N=0.3$ at $Z=0.02$

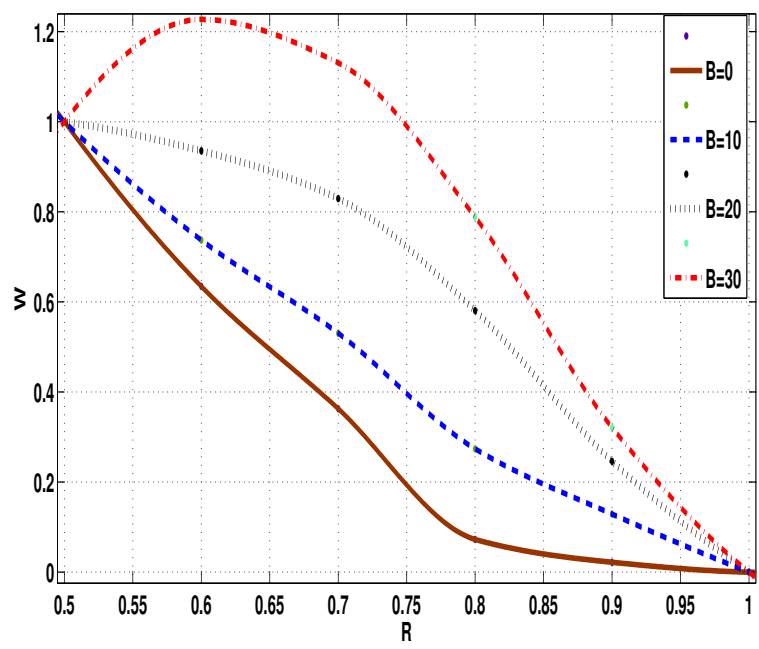

Figure 4. Tangential velocity profile for $N=0.5$ at $Z=0.02$

$U$ increases with the increase of Bingham number $B$ as well as aspect ratio $N$. For both stationary $(R t=0)$ as well as the rotating inner cylinder $(R t=20)$, it is observed that the velocity profile takes the parabolic form when Bingham number $B$ reaches zero (Newtonian fluid). However, the rotational effect on the axial velocity 


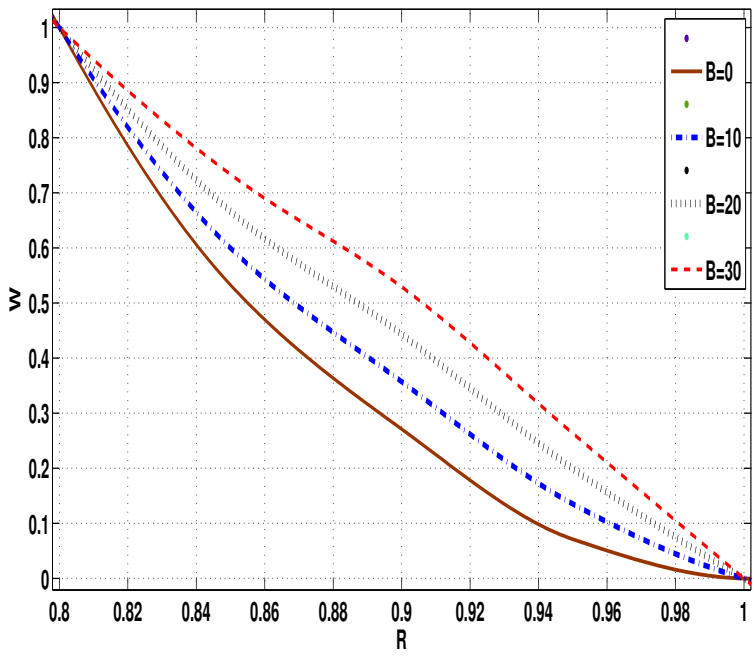

Figure 5. Tangential velocity profile for $N=0.8$ at $Z=0.02$

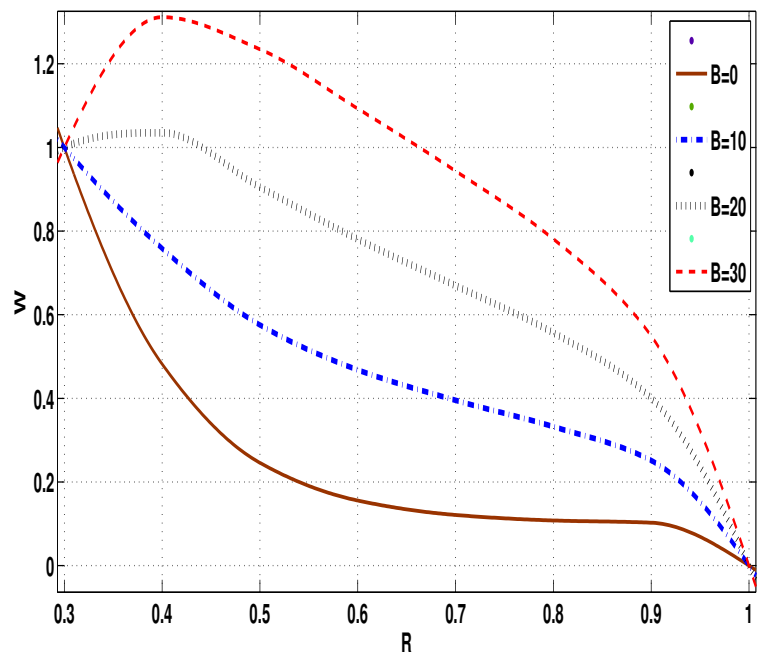

Figure 6 . Tangential velocity profile for $N=0.3$ at $Z=0.03$

component is very small as per the observed results. Here, our results corresponding to $B=0$ match with the results of Coney and El-Shaarawi 20 and $R t=0$ with various Bingham numbers match with the results of Kandasamy [7]. 


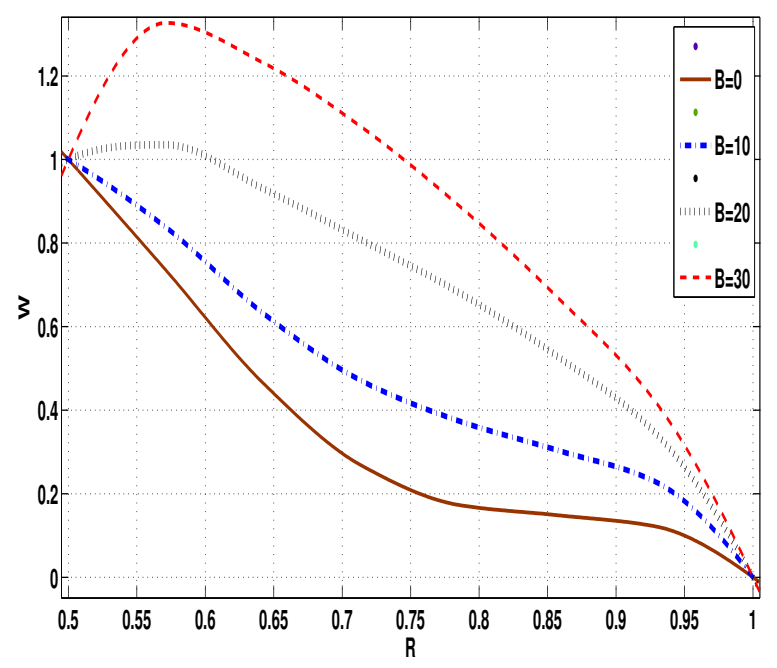

Figure 7. Tangential velocity profile for $N=0.5$ at $Z=0.03$

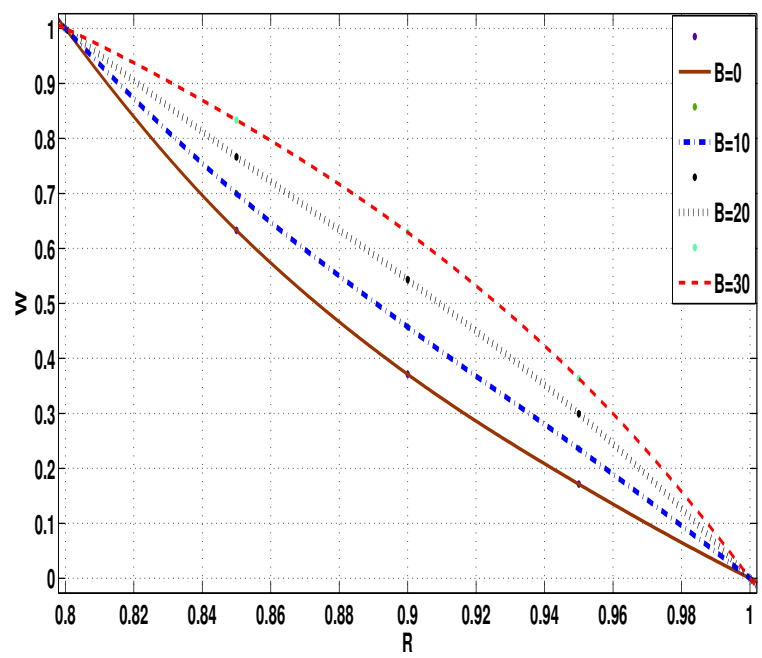

Figure 8. Tangential velocity profile for $N=0.8$ at $Z=0.03$

The radial velocity profile component $V$ for $N=0.3,0.5$ and 0.8 , for different values of Bingham number $B$ at axial positions of $Z=0.02,0.03$ are shown in Figures 15 to 20. Again, the values of the parameter $R t$ are taken as 0 and 20 for computational purposes. The values of radial velocity are negative in the region near the outer wall, 


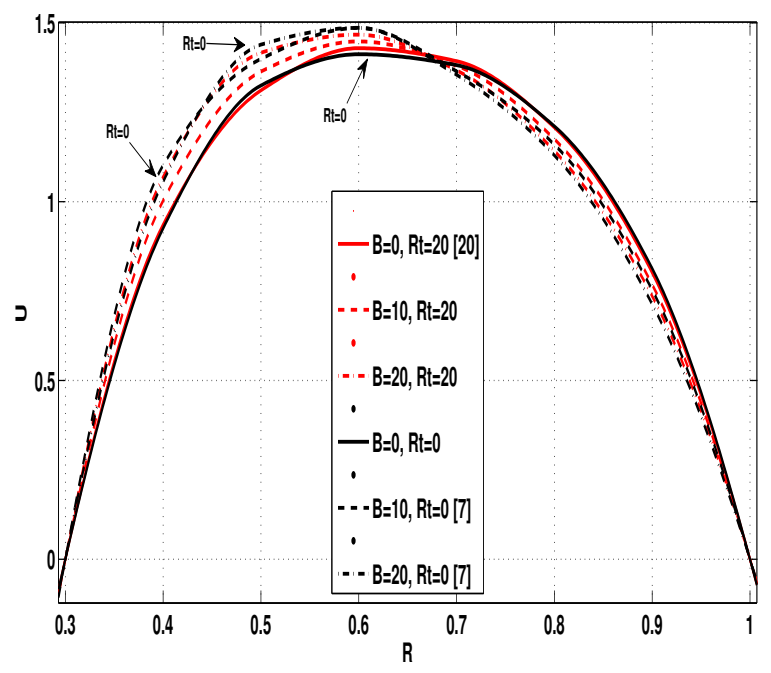

Figure 9. Axial velocity profile for $=0.3$ at $Z=0.02$

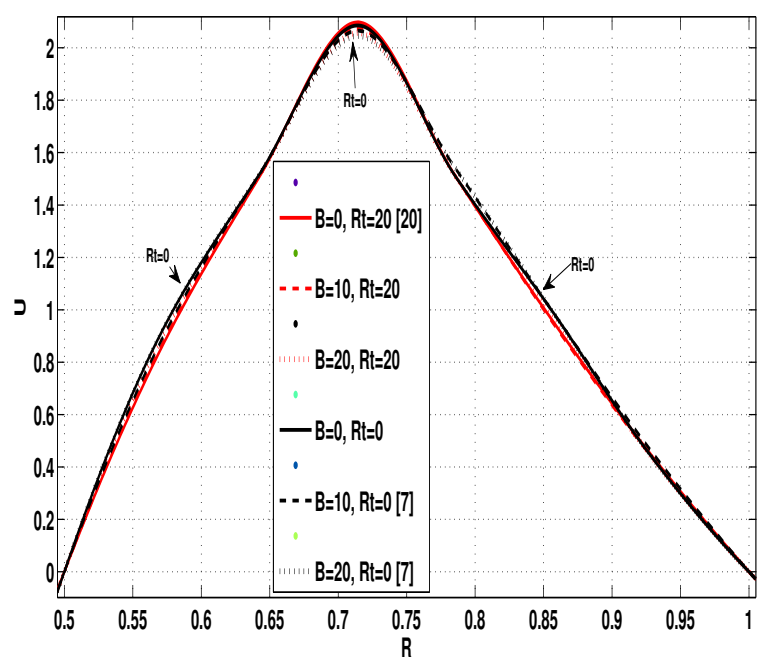

Figure 10. Axial velocity profile for $N=0.5$ at $Z=0.02$

since it is in the opposite direction to the radial coordinate $R$, and it has positive values near the inner wall because it has the same direction of the radial coordinate. The values of the radial velocity decreases with increase of Rt and Bingham number $B$ at any cross section of the axis. The results of particular cases like $R t=0$ (without 


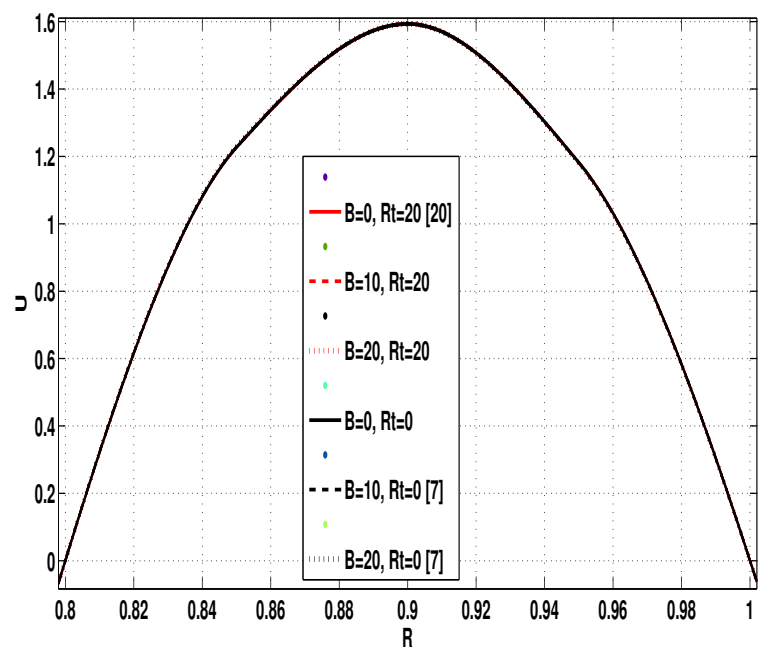

Figure 11. Axial velocity profile for $N=0.8$ at $Z=0.02$

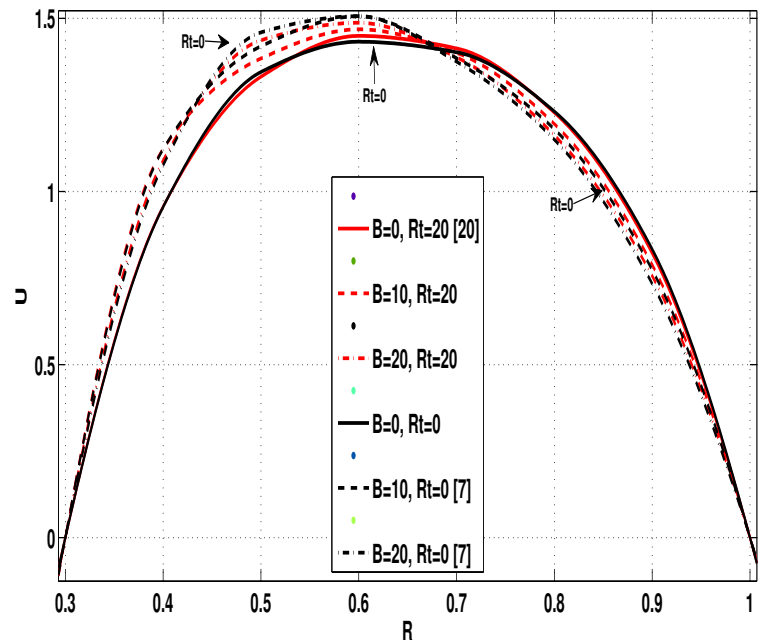

Figure 12. Axial velocity profile for $N=0.3$ at $Z=0.03$

rotation) and $B=0$ (Newtonian fluid) match fully with earlier research work [7, 20, respectively.

Figures 21 to 26 show the distribution of the pressure $P$ along the radial coordinate $R$ for the same chosen values of parameters. It is found that the value of $P$ increases 


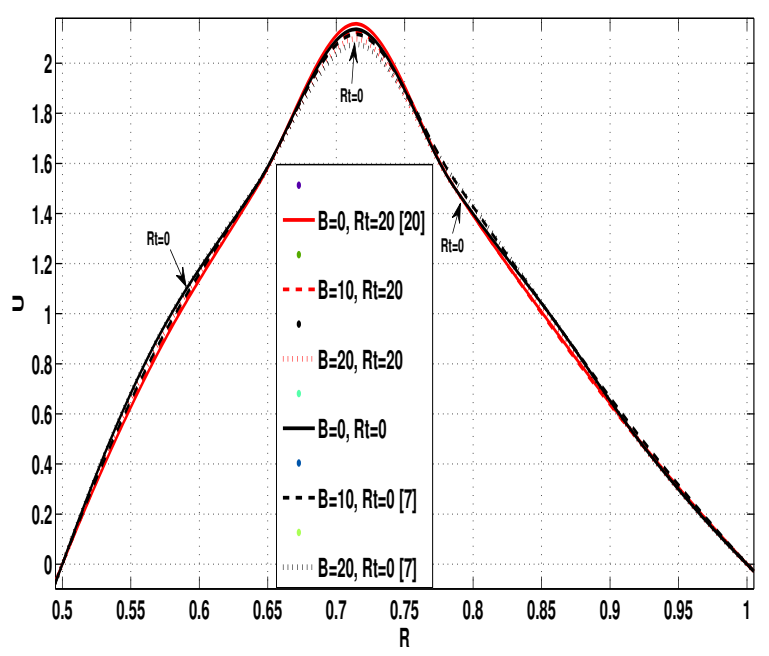

Figure 13. Axial velocity profile for $N=0.5$ at $Z=0.03$

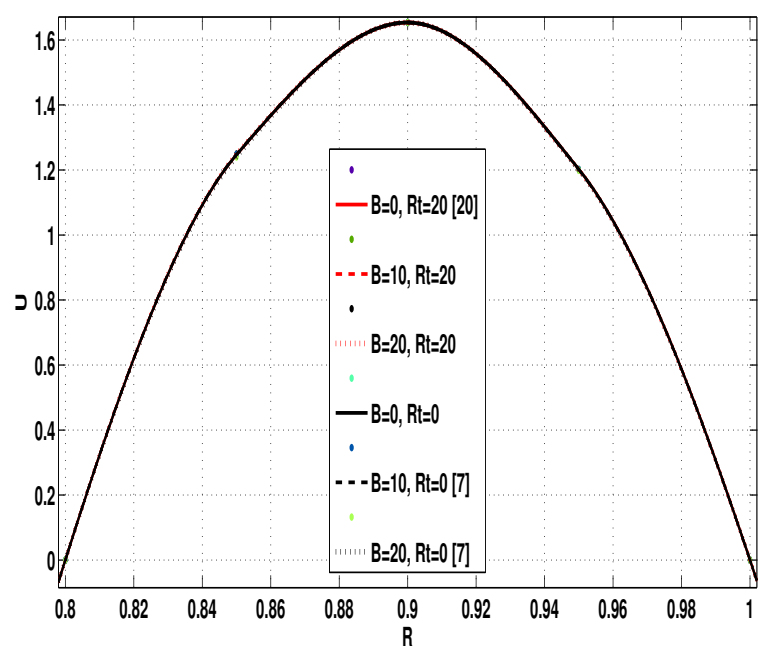

Figure 14. Axial velocity profile for $N=0.8$ at $Z=0.03$

from a minimum at the inner wall to a maximum at the outer wall. Further, it is found that with increase of Bingham numbers the pressure values $P$ increase. This is because the pressure will tend to be higher for thick viscous fluids. Moreover, it is observed that the pressure slowly becomes independent of the radial coordinate in the 


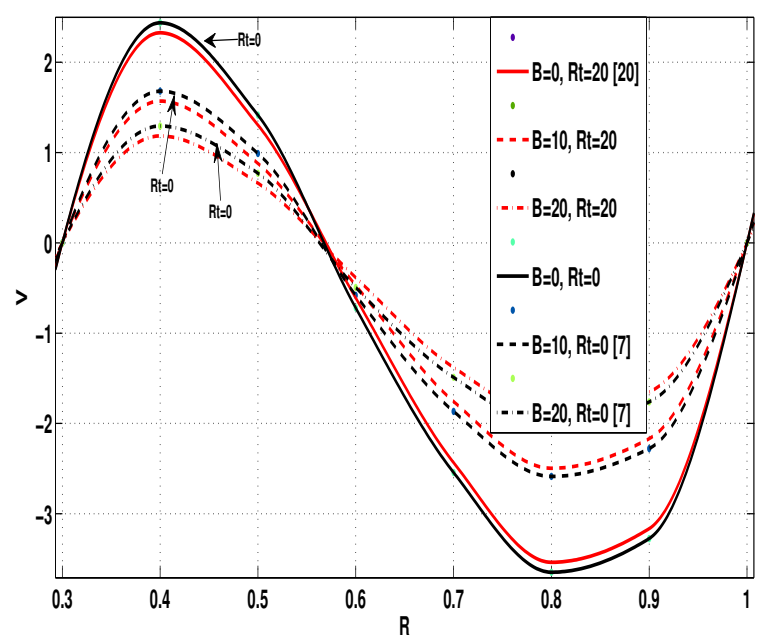

Figure 15. Radial velocity profile for $N=0.3$ at $Z=0.02$

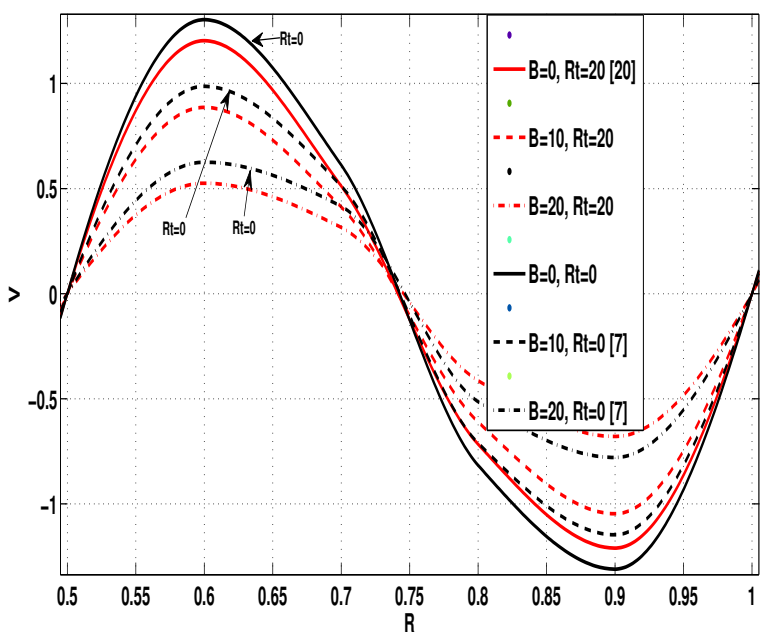

Figure 16. Radial velocity profile for $N=0.5$ at $Z=0.02$

region close to the outer wall. The effect of inner wall rotation on the fluid pressure seems to be very low at any cross section. Here also, the results corresponding to $B=0$ and $R t=0$ are in agreement with the earlier results [7, 20, respectively. 


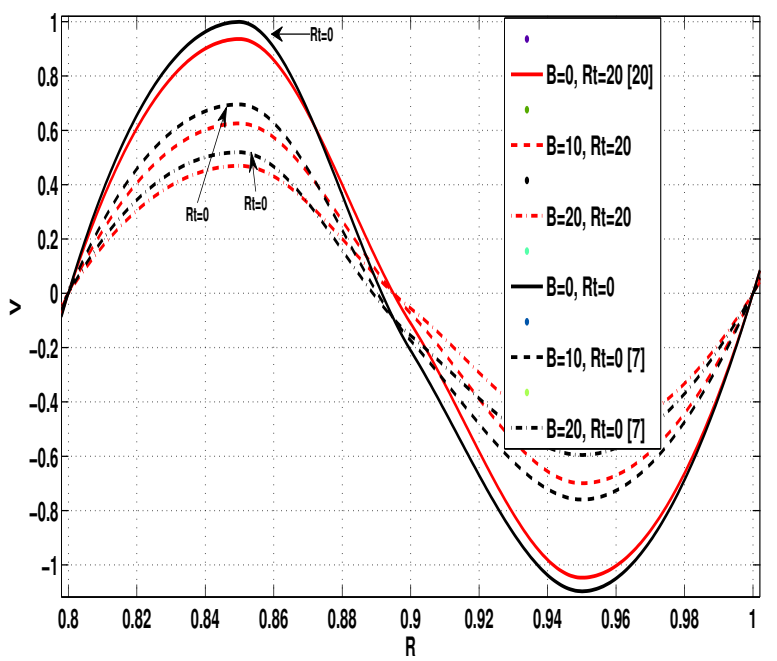

Figure 17. Radial velocity profile for $N=0.8$ at $Z=0.02$

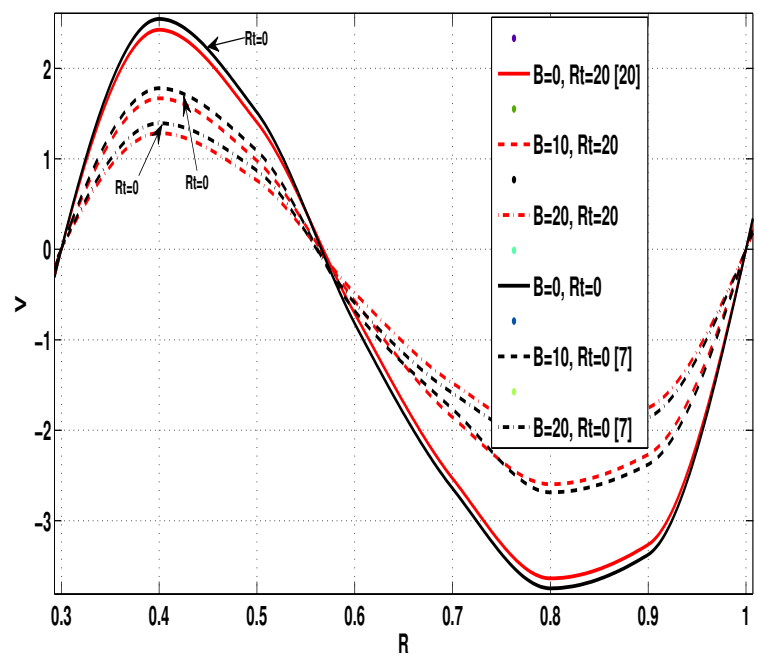

Figure 18. Radial velocity profile for $N=0.3$ at $Z=0.03$ 


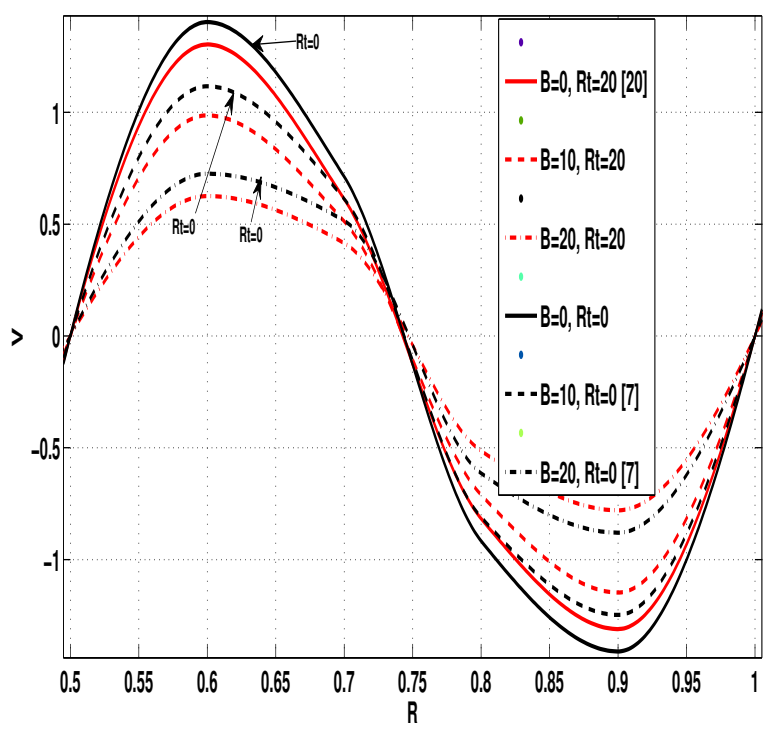

Figure 19. Radial velocity profile for $N=0.5$ at $Z=0.03$

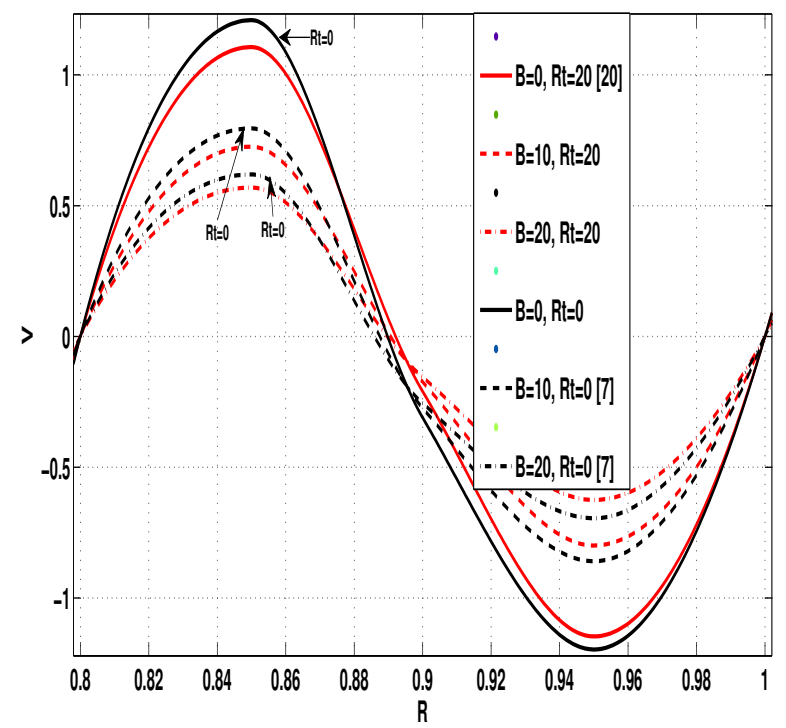

Figure 20. Radial velocity profile for $N=0.8$ at $Z=0.03$ 


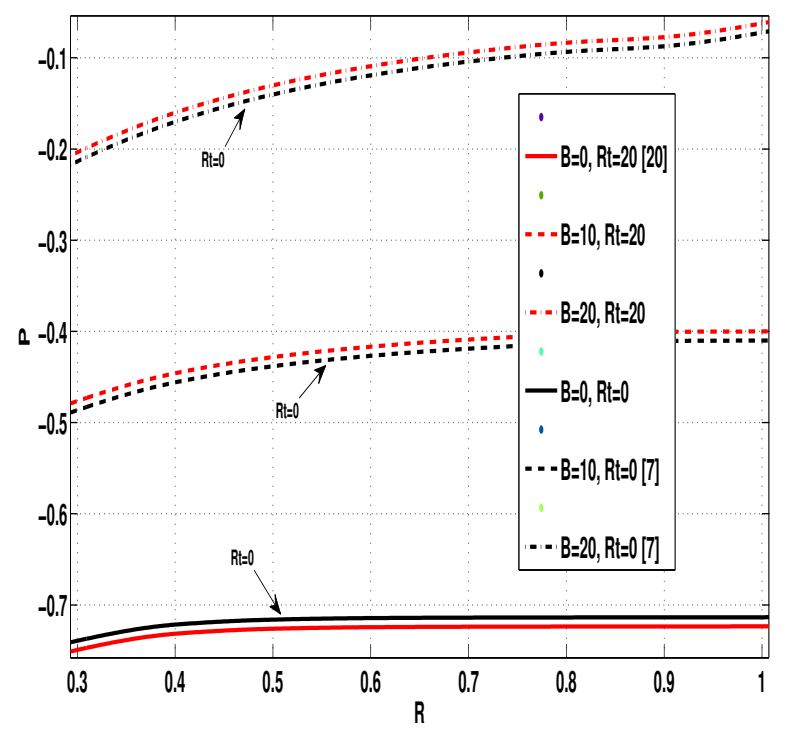

Figure 21. Pressure distribution for $N=0.3$ at $Z=0.02$

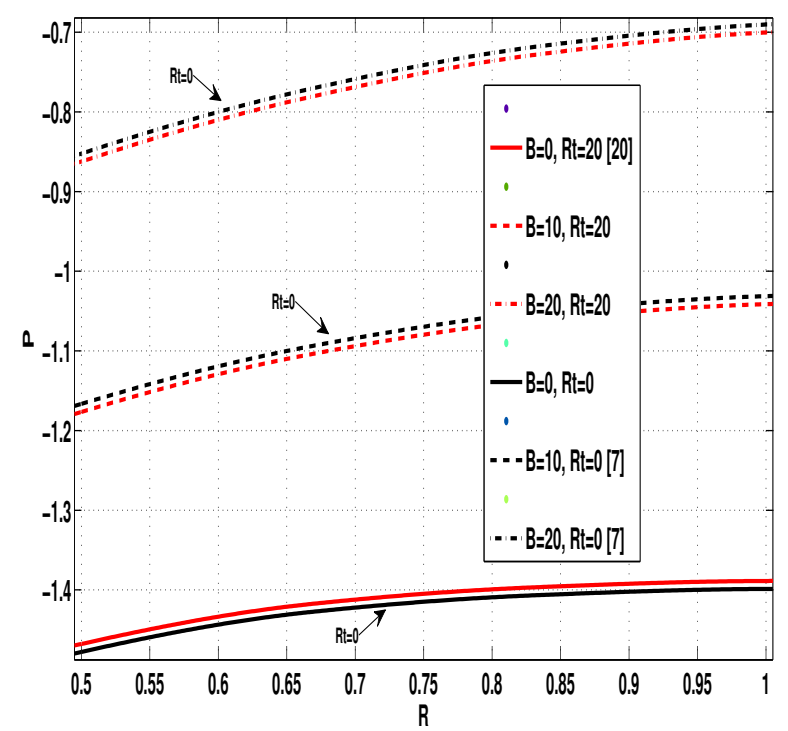

Figure 22. Pressure distribution for $N=0.5$ at $Z=0.02$ 


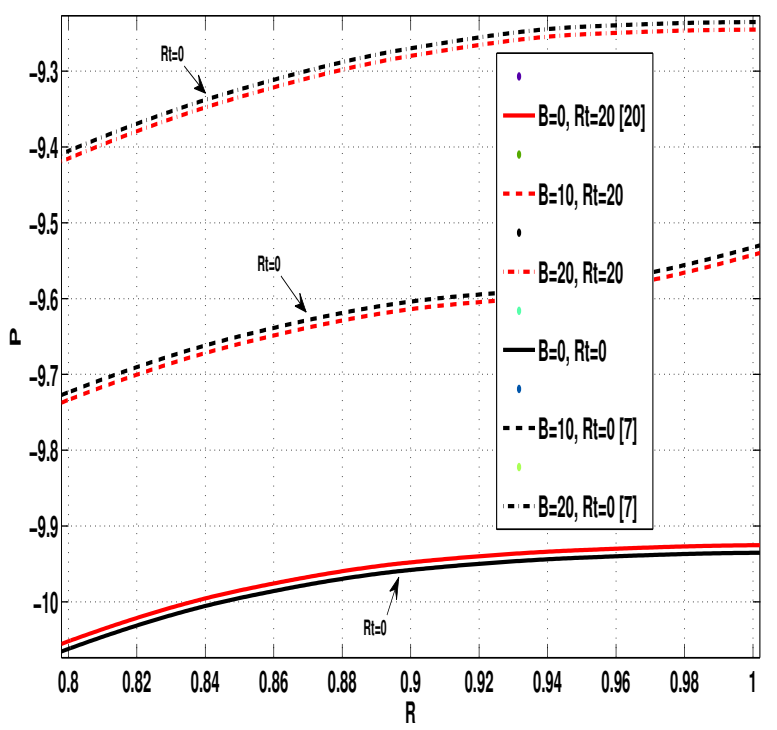

Figure 23. Pressure distribution for $N=0.8$ at $Z=0.02$

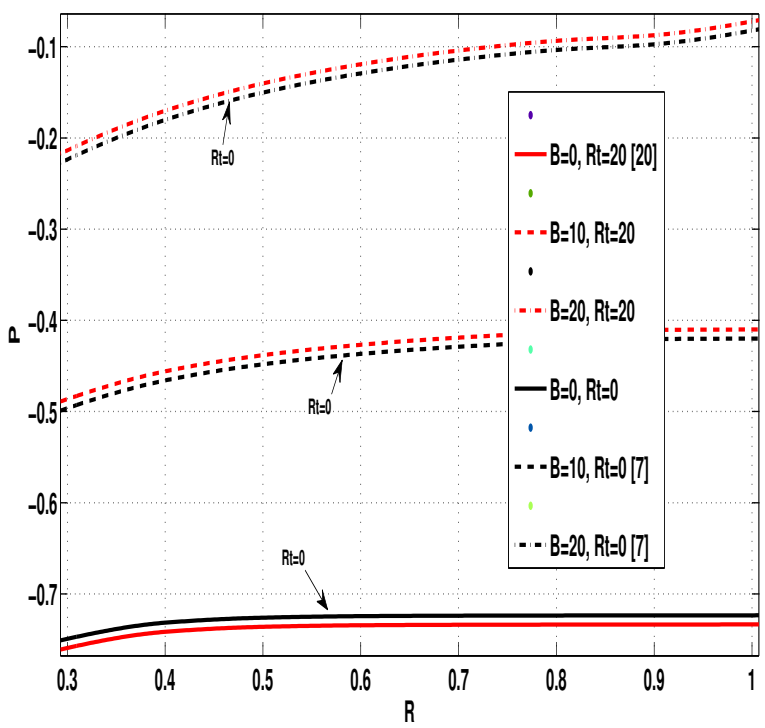

Figure 24. Pressure distribution for $N=0.3$ at $Z=0.03$ 


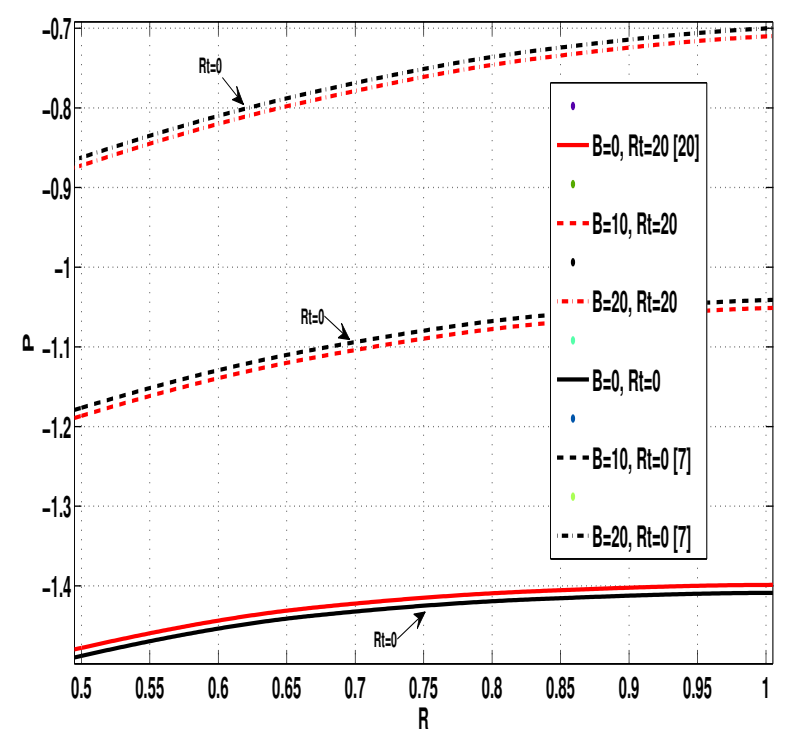

Figure 25. Pressure distribution for $N=0.5$ at $Z=0.03$

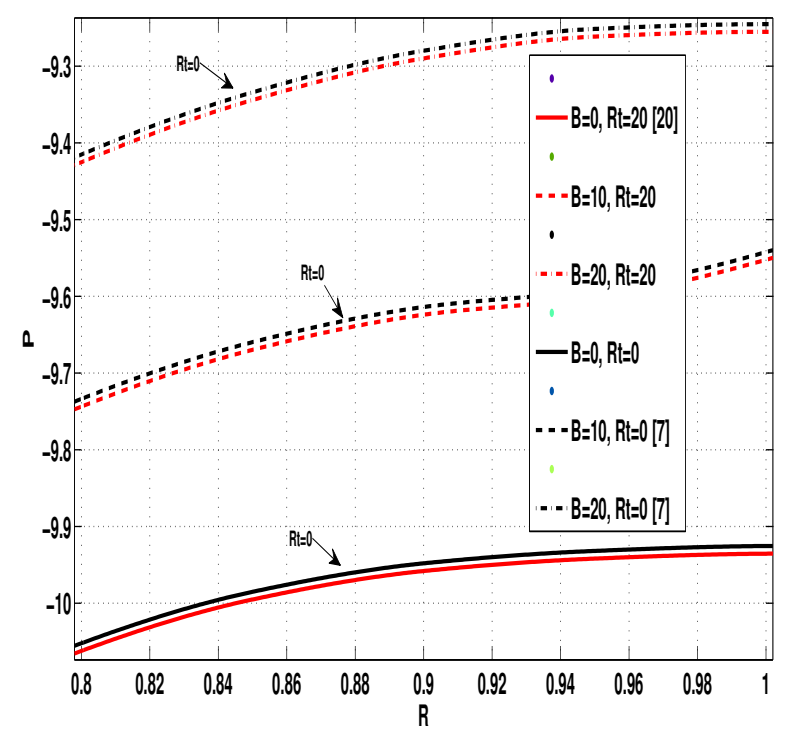

Figure 26. Pressure Distribution for $N=0.8$ at $Z=0.03$ 


\section{CONCLUSION}

Numerical results for the entrance region flow in concentric annuli with rotating inner wall for Bingham fluid were presented. The effects of the parameters $N, B$ and $R t$ on the pressure distribution, the velocity profiles are studied. Numerical calculations were performed for all admissible values of Bingham number $B$, aspect ratio $N$ and the parameter $R t$. The velocity distributions and pressure distribution along radial direction $R$ were presented geometrically. The present results are found to be in agreement with the results corresponding to various particular cases available in literature.

From this study, the following can be concluded.

1. Tangential velocity decreases from the inner wall to the outer wall of the annulus.

2. Increasing the aspect ratio $N$, the axial velocity component $U$ increases at all values of Bingham numbers $B$.

3. Radial velocity is found to be dependent only on the axial coordinate.

4. Pressure increases from a minimum at the inner wall to a maximum at the outer wall of the annulus and pressure does not vary much with respect to the radial coordinate in the region near the outer wall.

5. The effect of the inner wall rotation on all these flow characteristics seems to have little significance.

Acknowledgement. The authors would like to express our sincere gratitude to the reviewers and editor for their useful comments and suggestions which have helped the presentation of this work.

\section{REFERENCES}

1. Mishra, I. M., Surendra Kumar and Mishra, P.: Entrance region flow of Bingham plastic fluids in concentric annulus. Indian Journal of Technology, 23, (1985), 81-87.

2. Batra, R. L. and Bigyani, D.: Flow of a Casson fluid between two rotating cylinders. Fluid Dynamic Research, 9, (1992), 133-141.

3. Maia, M. C. A. and Gasparetto, C. A.: A numerical solution for entrance region of non-Newtonian flow in annuli. Brazilian Journal of Chemical Eng., 20, (2003), 201-211.

4. Sayed-Ahmed, M. E. and Hazem Sharaf-El-Din.: Entrance region flow of a powerlaw fluid in concentric annuli with rotating inner wall. International Communications in Heat and Mass Transfer, 33, (2006), 654-665.

5. Kandasamy, A. and SRinivasa, R. N.: Entrance region flow in concentric annuli with rotating inner wall for Herschel-Bulkley fluids. International Journal of Applied and Computational Mathematics, 1, (2015), 235-249.

6. Bird, R. D., Dai, G. C. and Yarusso, B. J.: The rheology and flow of viscoplastic materials. Reviews in Chemical Engineering, 1, (1982), 1-70.

7. Kandasamy, A.: Entrance region flow heat transfer in concentric annuli for a Bingham fluid. In Proceedings of Third Asian-Pacific Conference on Computational Mechanics, Seoul, Korea, 1996, pp. 1697-1702. 
8. Round, G. F. and YU. S.: Entrance laminar flows of viscoplastic fluids in concentric annuli. The Canadian Journal of Chemical Engineering, 71, (1993), 642-645.

9. Misra, J. C. and Ghosh, S. K.: Flow of a Casson fluid in a narrow tube with a side branch. International Journal of Engineering science, 38, (2000), 2045-2077.

10. Dash, R. K., Mehta, K. N. and Jayaram, G.: Casson fluid flow in a pipe filled with a homogeneous porous medium. International Journal of Engineering Science, 34, (1996), $1145-1156$.

11. Ahmed, M. E. S. and Atтia, H. A.: Magneto hydrodynamic flow and heat transfer of non-Newtonian fluid in an eccentric annulus. Canadian Journal of Physics, 76, (1998), 391-401.

12. Batra, R. L. and Bigyani Jena: Entrance region flow of blood in concentric annulus. International Journal of Engineering Science, 28, (1990), 407-419.

13. Manglik, R. and FAng, P.: Thermal processing of various non-Newtonian fluids in annular ducts. International Journal Heat and Mass Transfer, 45, (2002), 803-815.

14. Nouar, C., Lebouche, M., Devienne, R. and Riou, C.: Numerical analysis of the thermal convection for Herschel-Bulkley fluids. International Journal of Heat and Fluid Flow, 16, (1995), 223-232.

15. Poole, R. J. and Chhabra, R. P.: Development length requirements for fully developed Laminar pipe flow of yield stress fluids. Journal of Fluids Engineering, 132, (2010), 34501-34504.

16. Galanis, N. and Rashidi, M. M.: Entropy generation in non-Newtonian fluids due to heat and mass transfer in the entrance region of ducts. Heat Mass Transfer, 48, (2012), 1647-1662.

17. Rashidi, M. M., Rajvanshi, S. C., Kavyani, N., Keimanesh, M., Pop, I. and Saini, B. S.: Investigation of heat transfer in a porous annulus with pulsating pressure gradient by homotopy analysis method. Arabian Journal for Science and Engineering, 39, (2014), 5113-5128.

18. Rekha G. Pai and Kandasamy, A.: Entrance region flow of Bingham fluid in an annular cylinder. International Journal of Applied Engineering Research, 5, (2014), 70837101.

19. Schlichting, H. and Gersten, K.: Boundary Layer Theory. 8th ed., Springer, 2000.

20. Coney, J. E. R. and El-ShaArawi, M. A. I.: A contribution to the numerical solution of developing laminar flow in the entrance region of concentric annuli with rotating inner walls. Journal of Fluids Engineering, 96, (1974), 333-340. 